\title{
¿PUEDEN ADQUIRIR PALABRAS LOS ANIMALES? SOBRE EL APRENDIZAJE DE PALABRAS POR UN PERRO
}

\author{
Victor M. Longa y JuAn J. López Rivera \\ Universidad de Santiago de Compostela \\ fevlonga@usc.es, fejjrive@usc.es
}

\begin{abstract}
Resumen
Diferentes propuestas han atribuido equivalentes de palabras humanas a variadas especies animales desde las perspectivas de la producción o de la percepción. Este artículo discute críticamente tales propuestas, con especial atención a Kaminski, Call y Fisher (2004), que analizan las habilidades de 'aprendizaje de palabras' de un perro doméstico. Nuestro trabajo defiende que atribuir palabras (o elementos parecidos a ellas) a los animales supone malentender qué implican las palabras, al concebirlas de manera mucho más simple de lo que son realmente. Por tanto, intentamos mostrar que los aparentes éxitos léxicos del citado perro, llamado Rico (y los de otros animales) no suponen verdaderas palabras, sino etiquetas para objetos o conceptos.
\end{abstract}

PALABRAS CLAVE: cognición animal, adquisición animal de palabras, palabra, etiqueta, fast mapping.

\begin{abstract}
It has been argued that several animal species possess the equivalent of human words from the perspectives of production or perception. This article critically discusses such proposals, with special reference to Kaminski, Call \& Fisher's (2004) paper, which concentrates its work on the 'word learning' abilities of a domestic dog. We argue that attributing words (or word-like items) to animals supposes a misconception about what words actually imply. Such a misconception considers words to be much simpler than they are. Accordingly, the article suggests, and aims at showing, that the seemingly lexical achievements of the aforementioned dog, named Rico, (and those of other animals) are not with true words; instead, they are to be regarded as labels for objects or concepts.
\end{abstract}

KEY WORDS: animal cognition, animal word learning, word, label, fast mapping.

\section{Introducción}

Numerosos análisis del comportamiento comunicativo animal han atribuido capacidades lingüísticas de variado tipo (gramatical, semántico, léxico o pragmático) a numerosas especies animales. Por ejemplo, al respecto del componente gramatical, se han apreciado capacidades presintácticas en hormigas (Hölldobler y Wilson, 1994), pájaros (cfr. la 'sintaxis fonológica' de Marler, 1977) o gibones (Mitani y Marler, 1989). Por otro lado, también se han defendido capacidades sintácticas plenas, aunque simples, en ciertos animales; especialmente, en primates sometidos a entrenamiento (Fouts, 1997; Savage-Rumbaugh, Shanker y Taylor, 1998 o Rondal, 2000, por no citar los trabajos clásicos de Premack o los Gardner), pero también en monos en estado salvaje (Zuberbühler, 2002).

Queremos hacer constar nuestro agradecimiento al Dr. Euan MacPhail (Professor of Psychology, York University) por sus clarificadores comentarios sobre la noción de aprendizaje por exclusión. 
También se han atribuido capacidades léxicas a variadas especies. Según tal visión, animales humanos y no humanos divergirían sobre todo en el número de palabras poseídas o manejadas, pero no en la naturaleza de tales palabras. Es obvio que las limitaciones fisiológicas del tracto vocal de los animales impiden que pronuncien palabras humanas (excepto en algunos casos de imitación, como en loros; cfr. Patterson y Pepperberg, 1994, 1998, sobre la habilidad de los loros grises para producir pares mínimos fonéticamente distintos). Sin embargo, tales propuestas defienden que algunos animales pueden manejar palabras desde la perspectiva de la percepción, mientras que otros poseen equivalentes de palabras desde la de la producción, bien en modalidad oral (como ciertas vocalizaciones; cfr. infra) o bien en otras diferentes (signos manuales, fichas de plástico, etc.). De ahí que, según Lieberman (2003: 256), la idea de que sólo los seres humanos pueden poseer y usar palabras es "refuted by comparative studies of the communicative and linguistic abilities of dogs, apes, birds, and other species".

Las supuestas capacidades léxicas han sido defendidas en dos diferentes contextos: posesión natural por parte de animales salvajes (el ejemplo más conocido se refiere a las llamadas de alarma de los vervets; cfr. Seyfarth, Cheney y Marler, 1980a, 1980b) y, sobre todo, adquisición por parte de animales entrenados (sobre los delfines, cfr. Herman et alii, 1984; sobre monos, cfr. Premack, 1970 como estudio clásico sobre la adquisición de la 'función palabra'; cfr. Savage-Rumbaugh et alii, 1998 para una visión actual). En este último contexto, la mayor parte de estudios se han dedicado al entrenamiento de chimpancés. Tales esfuerzos han mostrado supuestamente que estos animales "can acquire about 150 words and devise new words as well as modifying the meaning of words that they already have" (Lieberman, 2003: 257). Por ello, según este autor, la capacidad léxica (también la sintáctica) es un rasgo primitivo (esto es, compartido con otras especies), y no derivado (únicamente humano).

Muy recientemente, se ha publicado un estudio que sugiere capacidades léxicas en una especie no muy investigada en el citado sentido hasta este momento. Kaminski, Call y Fisher (2004) nos ofrecen el caso de Rico, un perro doméstico, que puede asociar 'palabras' con objetos y retener tales asociaciones en la memoria. Este 'aprendizaje de palabras' derivaría del mecanismo de fast mapping (Carey, 1978; cfr. infra). El trabajo de Kaminski et alii (2004) es ciertamente muy relevante, y abre grandes horizontes sobre la especie investigada: como señalan los propios autores (Kaminski et alii, 2004: 1682), el 'tamaño del vocabulario' de Rico es mucho mayor del constatado en los experimentos realizados previamente con perros. De hecho, los resultados obtenidos por Rico, resumidos en la adquisición de unos 200 elementos, igualan (o incluso sobrepasan) el conocimiento léxico alcanzado por los primates. Además, debe notarse, como afirma Bloom (2004: 1605), que los chimpancés nunca han mostrado de manera concluyente el fast mapping que Rico parece poseer (aunque los tests de Premack sobre la chimpancé Sarah parecían arrojar alguna prueba de su existencia, no llegaron a ser estadísticamente significativos).

El presente artículo analiza críticamente la atribución de palabras a animales. Más concretamente, discutirá la supuesta habilidad léxica de Rico, pero tratará de proyectar las conclusiones obtenidas a la cuestión de la capacidad léxica animal, natural o entrenada. A nuestro juicio, la asunción de que Rico (u otros animales) puede aprender palabras supone malentender las implicaciones de la noción de palabra, de modo que esta unidad es considerada como algo mucho más simple de lo que es realmente, reduciéndola a una mera aso- 
ciación estímulo-objeto. De hecho, es muy revelador en este sentido, a la par que sorprendente, que Kaminski et alii (2004) usen las nociones de palabra, etiqueta y nombre para un objeto de modo plenamente intercambiable entre sí (cfr. nota 2). Nuestro trabajo considera que los aparentes éxitos léxicos de Rico y de otros animales no suponen el aprendizaje de verdaderas palabras, sino el de etiquetas o nombres para objetos. No es nuestra intención poner en duda el mecanismo de fast mapping en Rico, cuya posesión es en sí misma muy relevante, sino cuestionar cómo funciona en relación a la adquisición de palabras: aunque el fast mapping es un mecanismo necesario para adquirir palabras, no es en sí mismo suficiente para lograr tal objetivo.

Es obvio que nuestra postura no implica la adopción del progresivismo, perspectiva que considera "the perfection of the human mind and brain to be the culmination of evolution" (Downes, 2003: 37). Por el contrario, asumimos que la 'estupidez subyacente' de todos los procesos evolutivos (Dennett, 1995: 72) originó numerosos sistemas comunicativos (uno de ellos, el lenguaje humano) que carecen de cualquier rastro de direccionalidad. Desde esta perspectiva, no es sorprendente que las palabras sean específicas de nuestra especie, de igual modo que otras especies poseen comportamientos comunicativos complejos que son únicamente suyos.

La estructura del trabajo es la siguiente: el apartado 2 expone brevemente el marco de Kaminski et alii (2004), sus resultados y asunciones. Por su parte, el 3 analiza la noción de palabra y sus grandes diferencias con respecto a la de etiqueta, defendiendo que Rico y otros animales no adquieren palabras, sino etiquetas. Además, discute la relación entre fast mapping y dominio de las palabras. Por último, el 4 ofrece las principales conclusiones.

\section{EI 'aprendizaje de palabras' de Rico: breve descripción del procedimiento}

Esta sección presentará los principales aspectos del caso de Rico (para detalles adicionales, cfr. Kaminski et alii 2004). El objetivo del trabajo citado es investigar el 'aprendizaje de palabras' ("ability to acquire the relation between a word and the object that this word refers to; Kaminski et alii, 2004: 1682) de Rico, un collie de la frontera nacido en 1994 que vive como un perro doméstico. Sus dueños informaron de que Rico conocía las etiquetas de unos 200 elementos, que podía recoger y entregar a petición de ellos. Esta habilidad, desarrollada mediante entrenamiento, empezó cuando Rico tenía 10 meses. El entrenamiento se basaba en presentar periódicamente nuevos elementos a Rico, definirlos por la palabra que les corresponde y repetir esa palabra dos o tres veces. Rico era recompensado si cobraba el elemento correcto.

A partir de esta situación, Kaminski et alii (2004) investigaron fundamentalmente dos aspectos. En primer lugar, evaluaron bajo condiciones controladas la habilidad de Rico para recuperar diferentes objetos: Rico recuperó correctamente 37 de los 40 objetos reclamados. En segundo lugar, analizaron su habilidad de fast mapping, esto es, su habilidad para establecer nuevas asociaciones entre palabra y objeto sobre la base de una única exposición, y para retener tales asociaciones en el tiempo. El procedimiento era el siguiente: en una habitación adyacente a aquella donde estaban Rico y sus dueños, se colocó un nuevo objeto, junto con otros 7 objetos familiares. En el primer ensayo de cada sesión, los dueños pedían a Rico que trajera un elemento ya conocido. Durante el segundo o tercer ensayo, se le pedía que trajera el nuevo elemento, usando para ello la palabra que designaba ese nuevo objeto. 
Este procedimiento fue repetido durante 10 sesiones, en las que se introdujeron 10 nuevos objetos. Rico identificó y cobró el nuevo elemento en 7 de las 10 sesiones. Según Kaminski et alii (2004), esto sugiere que Rico infería las palabras de los nuevos elementos mediante un aprendizaje por exclusión, de modo que adquirió el principio de que "objects have labels" ${ }^{2}$. Cuatro semanas después de los experimentos, se sometió a prueba la retención por parte de Rico de las asociaciones establecidas entre las nuevas palabras y los nuevos objetos, consiguiendo traer los elementos solicitados en 3 de las 6 sesiones.

Aunque Kaminski et alii (2004) no niegan que los niños tienen un conocimiento mucho mayor y más profundo del significado de las palabras que el mostrado por Rico, lo cual se aprecia en aspectos como el tamaño del vocabulario, el uso productivo de nuevas palabras, o la habilidad para diferenciar entre tipos de palabras, señalan sin embargo que "These experiments demonstrate that Rico reliably associates arbitrary acoustic patterns (human words) with specific items in his environment" (Kaminski et alii 2004: 1683). Con su estudio, los autores pretenden explorar la naturaleza de los principios que rigen el aprendizaje de palabras: a su juicio, el caso de Rico muestra que esos principios no son específicamente humanos, sino compartidos con otras especies. Más concretamente, Kaminski et alii (2004: 1683) apuntan que las habilidades de Rico se pueden descomponer en tres mecanismos más simples: (1) adquisición del principio de que los objetos tienen etiquetas; (2) aprendizaje por exclusión; y (3) habilidad para almacenar el conocimiento alcanzado en la memoria. Por tanto, según Kaminski et alii (2004: 1683), los resultados de su estudio

strongly support the view that a seemingly complex linguistic skill previously described only in human children may be mediated by simpler cognitive building blocks that are also present in another species.

Es obvio que la clave del problema se basa en la noción de palabra asumida por esos autores, dado que igualan las nociones de palabra, etiqueta y nombre de una cosa (cfr. nota 2). Sin embargo, desde una perspectiva lingüística (y filosófica), tales nociones no son equivalentes: poseer etiquetas (o nombres) no es lo mismo que poseer palabras. Por ello, sostendremos que los tres principios sugeridos por los autores no garantizan que Rico posea palabras. Aunque es cierto que compartimos habilidades con los animales (especialmente, con mamíferos y aves) que son relevantes para la adquisición y el uso de palabras, tales habilidades, representadas por los tres principios citados, son necesarias, pero no suficientes, para poseer palabras. Por ello, no son concluyentes para determinar que Rico tiene capacidades léxicas.

\section{Discusión}

\subsection{Palabras y etiquetas: enfoque preliminar}

Como señalamos antes, aunque Kaminski et alii (2004) reconocen obvias diferencias entre la capacidad de un niño para adquirir palabras y la de Rico, no es menos cierto que

2 Nótese que Kaminski et alii (2004) utilizan en ocasiones el término 'nombre' para aludir a la palabra que corresponde a un objeto, mientras que otras veces usan 'etiqueta' con el mismo propósito. De ahí que, como señalamos antes, igualen las nociones de 'palabra', 'nombre' y 'etiqueta'. 
igualan el resultado final de la adquisición, que sería en ambos casos la asociación entre un arbitrary acoustic pattern y un objeto específico. Es decir, este modelo acústico arbitrario vinculado directamente a un objeto sería descrito, en el sentido más amplio, como una palabra, etiqueta o nombre para un objeto. Esta perspectiva manejada por Kaminski et alii deriva de su aceptación de la concepción no técnica sobre las palabras, que las entiende como meras etiquetas o nombres para un elemento. La base de la citada concepción reside en la aparente naturaleza muy simple del aprendizaje (y uso) de palabras, "at least to scholars who are not directly engaged in its study" (Bloom, 2000: 3). De este modo, tal adquisición parece implicar "merely the memorization of a series of paired associates: dog refers to dog, water refers to water, Mommy refers to Mommy, and so on" (Bloom, 2000: 1) $)^{3}$.

Por tanto, según la citada concepción no técnica o popular, palabras, etiquetas y nombres para objetos responderían supuestamente a un trazado directo (cfr. la cita de la nota 3 ), donde el objeto es automáticamente evocado por la palabra, la etiqueta o el nombre de ese objeto. Si bien esta hipótesis puede explicar la naturaleza de las etiquetas (y de los nombres de los objetos), no puede dar cuenta de la de las palabras. Desde una perspectiva instrumental o funcional, tanto palabras como etiquetas pueden usarse para objetos específicos: las palabras 'hablan' sobre los objetos en contextos comunicativos concretos, pudiendo así situarlos e individualizarlos dentro de un contexto de referencia. Sin embargo, el poder de las palabras va mucho más allá, dado que muchas palabras se refieren a objetos irreales, que carecen de cualquier referencia en el mundo. Frente a ello, las etiquetas se restringen a la realidad (cfr. 3.2.). Por esta razón, la adquisición de palabras sobrepasa con mucho una simple asociación entre un modelo acústico (o manual) arbitrario y un objeto concreto.

Tal idea en realidad no es nueva en lingüística. Hace casi 100 años, Saussure criticó la asunción de que las lenguas son meras nomenclaturas, listas de términos que corresponden a listas de cosas. Su crítica trataba así de romper con la concepción según la que los elementos lingüísticos eran meras etiquetas o nombres para cosas, postura que Saussure (1916: 101) consideraba simplista. Con sus palabras: "El signo lingüístico une no una cosa y un nombre, sino un concepto y una imagen acústica" (Saussure, 1916: 102) 4 . La imagen acústica "no es el sonido material, cosa puramente física, sino la huella psíquica de ese sonido, la representación que de él nos da el testimonio de nuestros sentidos" (Saussure, 1916: 102). Por tanto, entre el sonido material que es la evidencia de la palabra y el objeto al que se refiere el sonido existen dos entidades de naturaleza psicológica que rompen el vínculo directo palabra-objeto. Dada la existencia de esas dos entidades (especialmente, de

3 También Tomasello (2003: 43) caracteriza de manera nítida, rechazándola, la supuesta simplicidad del aprendizaje de palabras: "Word learning is often characterized as a kind of mini-linguistics lesson, similar to vocabulary lessons in a foreign language classroom, in which adults point to and name objects for children. In this pointingand-naming game, the process seems relatively simple. The child only has to associate the word she is currently hearing with the thing she is currently seeing. Another popular metaphor is that children 'map' words onto things (or perhaps concepts of things). Unfortunately, this game is not representative of the vast majority of word-learning situations that children encounter in their daily lives".

4 Según Saussure (1916: 103), "Llamamos signo a la combinación del concepto y de la imagen acústica: pero en el uso corriente este término designa, generalmente, a la imagen acústica solo, por ejemplo, una palabra (arbor, etc.)". Y sigue señalando que "Se olvida que si arbor es llamado signo, es solo porque lleva en sí el concepto «árbol», de tal suerte que la idea de la parte sensorial implica la de la totalidad". Dados los rasgos que definen una palabra (cfr. infra), así como los propios comentarios de Saussure, las palabras satisfacen todos los requisitos para ser consideradas signos. Tal aspecto ha quedado bien asentado en el pensamiento lingüístico posterior. 
la conceptual), las palabras trabajan mediante operaciones que representan mucho más que un mero etiquetado de un objeto.

Varias propuestas han considerado que algunas vocalizaciones animales son similares a las palabras humanas. Por ejemplo, según Rondal (2000: 31), algunas de tales vocalizaciones tienen el estatus de signos o protosignos, compartiendo así un rasgo fundamental de las palabras. Sin embargo, a nuestro juicio la similitud no puede sostenerse: las vocalizaciones son etiquetas (como las aparentes palabras con las que trabaja Rico), pero no verdaderas palabras en el sentido técnico, ni equivalentes de ellas. Para ello, analizaremos brevemente el caso más representativo de supuesto parecido de vocalizaciones animales con las palabras, el sistema de llamadas de alarma de los monos vervet (Cercophitecus aethiops).

Este sistema, compuesto por tres diferentes llamadas, alerta de la presencia de tres tipos de predadores: águilas, serpientes y leopardos. Cada llamada provoca una huida diferente, que pueda contrarrestar eficazmente las características de cada predador: la llamada para águila hace que los monos bajen de los árboles y se escondan entre los arbustos, mientras que la llamada para leopardo provoca que suban a las ramas más altas de los árboles; finalmente, al oír la llamada para serpiente, observan el suelo, adoptando normalmente una postura erecta, y acosan en grupo al ofidio. Tales llamadas se han apreciado como uno de los escasos ejemplos de comunicación referencial animal (no ligada a emociones), por lo que han sido consideradas como equivalentes de las palabras 'águila', 'leopardo' y 'serpiente' (Seyfarth, Cheney y Marler, 1980a, 1980b; cfr. también Cheney y Seyfarth, 1990).

Según Seyfarth et alii (1980a, 1980b), las llamadas de los vervets poseen un rasgo central del lenguaje: sonidos arbitrarios con contenido referencial. Esta arbitrariedad se combina con otros rasgos también compartidos por las palabras: la propia llamada especifica el referente, sin necesidad de ver al predador ni al mono que la emite; esto es, el sonido es suficiente si se conoce el código ${ }^{6}$ (nótese que ambos rasgos pueden atribuirse a Rico desde la perspectiva del oyente, en tanto que el perro entiende sonidos arbitrarios referenciales en ausencia del referente físico).

5 Téngase en cuenta que las llamadas animales de alarma no se restringen a los vervets: de hecho, numerosas especies (como pollos, ardillas, o varias especies de pájaros) las emplean. Sin embargo, como señala Tomasello (2003: 10), "no one considers them to be symbolic or referential in a human-like way". De todos modos, debe hacerse notar que la noción de referencialidad es utilizada de manera diferente según el dominio en que se inscriba la investigación, como muestra claramente Pepperberg (1999: cap. 3) en su discusión de qué es una verdadera comunicación referencial: así por ejemplo, señala Pepperberg (1999: 42-44) que los biólogos suelen emplear la noción de referencialidad en el sentido contextual, de modo que "An animal uses a symbol to indicate the presence of, or to request, a particular type of item or a class of such items": este sentido es precisamente el empleado por Seyfarth et alii (1980a, 1980b), o por Evans y Evans (1999), quienes defienden la supuesta naturaleza referencial de las llamadas de los pollos que indican comida. Frente a este uso, la referencialidad en sentido lingüistico requiere "full abstract use of a symbol".

6 No obstante, trabajos recientes de estos autores han suavizado en alguna medida sus afirmaciones previas: aunque Cheney y Seyfarth (1999: 639-640) señalan que, desde la perspectiva del receptor, tales llamadas de alarma presentan aspectos comunes con las señales semánticas humanas, desde la del emisor carecen de un aspecto central del lenguaje: parecen no tener en cuenta los estados mentales de la audiencia (como conocimiento, ignorancia, etc.). De hecho, si esos estados mentales fueran considerados, los adultos deberían corregir a los jóvenes cuando estos últimos emiten una llamada equivocada, o bien la llamada de alarma debería inhibirse en aquellas situaciones en las que el resto del grupo ya es consciente del peligro. Sin embargo, ninguna de las dos situaciones se produce, por lo que no parece compartirse intencionalmente la comunicación. 
Sin embargo, ¿son las llamadas de los vervets verdaderos equivalentes de las palabras? Creemos que no, como se discutirá en el apartado 3.2. En todo caso, los rasgos de tales llamadas muestran que los animales no son meros autómatas, esto es, que no hay un trazado directo entre el input y el output. De hecho, según Bickerton (1990: 31) no puede existir relación directa entre llamada y objeto, dado que los errores que a veces cometen los vervets sugieren que el mono "está respondiendo a su propio acto de identificación, más que al objeto en sí mismo". Por tanto, el mono debe poseer algún tipo de concepto (evocado internamente) que interviene entre su percepción y su respuesta. Esto no debería sorprender, pues concuerda con el importante conocimiento alcanzado sobre la mente animal en las últimas décadas. Muchos estudios han mostrado que los animales no humanos poseen capacidades mentales altamente complejas y sofisticadas, como sentido numérico, navegación espacial, mapas mentales, relaciones geométricas y sociales, o reconocimiento sofisticado de objetos y seres, entre otros (cfr. las panorámicas de Budiansky, 1998, Hauser, 2000 o Spelke, 2003; sobre la cognición primate, cfr. Tomasello y Call, 1997; sobre la de aves, cfr. Pepperberg, 1999, 2004). Por ello, sobre la base de tales capacidades, es posible sostener, como hacen Hauser, Chomsky y Fitch (2002: 1575), que los animales "have rich conceptual representations" (si bien raramente se plasman en el terreno comunicativo).

Sin embargo, volviendo a las llamadas de alarma, el concepto del mono (como reacción a la alarma) produce una respuesta funcional concreta, la única posible: la de escapar. En otras palabras, la llamada se relaciona con un propósito específico. Por ello, parece que el mono identifica la llamada (y el concepto pertinente, por ejemplo 'águila') con el propio peligro.

Algo muy similar puede aplicarse a Rico: podría sostenerse la existencia de algún tipo de concepto en él del objeto evocado por la palabra, lo que conduciría a postular una relación indirecta entre los objetos recogidos por Rico y las 'palabras', etiquetas o nombres que provocan la tarea de recogida. Por tanto, esa relación sería mediada por un concepto. También se podría sostener que Rico responde exitosamente a actos de identificación, al ser capaz de recoger los objetos evocados por las 'palabras', etiquetas o nombres que pronuncian sus dueños. Y, finalmente, esos conceptos causan en Rico, como en los vervets, una respuesta funcional con un propósito específico, el de recoger objetos. Rico estaría así identificando cada 'palabra', etiqueta o nombre, y el concepto para el objeto, con la tarea de recogida de objetos (cfr. Bloom, 2004: 1605). Hemos comparado las llamadas de los vervets con las palabras usadas ante Rico no porque impliquen procesos semejantes (ni de aprendizaje ni de

Por otro lado, el análisis de Seyfarth et alii (1980a, 1980b) se basa en la visión usual sobre la comunicación animal, vinculada directamente con el intercambio de información. Sin embargo, el modelo de assessmentmanagement de Owings y Morton (1998) sugiere repensar drásticamente tal visión: según el citado modelo, la comunicación no supone intercambiar información (aspectos semánticos), sino manipular el comportamiento de los congéneres desde la perspectiva egoista del propio interés. Desde esta perspectiva, por tanto, las llamadas de los vervets serían meros productos inconscientes de la selección natural, en vez de un intercambio de información al uso: por ejemplo, el mono que alerta de la presencia de un leopardo o de un águila tiene el beneficio egoísta de provocar una estampida del resto de los miembros del grupo, estampida que desvía la atención con respecto al mono que avisó al grupo; téngase en cuenta que si un mono se limitara a escapar sin alertar al resto, el hecho de ser el único que corre lo haría muy vulnerable. En la medida en que el marco de Owings y Morton sea correcto, la supuesta naturaleza semántica de las llamadas de alarma quedaría seriamente cuestionada, o incluso restringida a la nada. 
utilización por parte o en presencia de estos animales), sino porque el resultado en ambos casos es funcional, ligado a una tarea específica.

\subsection{Palabras, percepción y realidad}

Aunque tanto las palabras como las llamadas de alarma presuponen conceptos que median entre ambas y sus referentes, la naturaleza y el uso de las palabras es muy diferente de la naturaleza y uso de las llamadas y de las 'palabras' aprendidas por Rico. La razón consiste en que las representaciones conceptuales humanas deben ser trazadas en representaciones lingüísticas, lo cual motiva que las palabras no se relacionen con propósitos específicos: su emisión no provoca la huida (como en los vervets) ni la recuperación de objetos (como en Rico). Las palabras posibilitan disociar el concepto correspondiente a un animal peligroso del propio concepto de peligro, del mismo modo en que disociamos los conceptos que usamos cuando nos referimos a objetos de la tarea de búsqueda y recogida de tales objetos ${ }^{7}$.

Mientras las llamadas de alarma evocan automáticamente un concepto de un animal asociado con el peligro que supone para los monos, y las 'palabras' dirigidas a Rico evocan también automáticamente un concepto de un objeto relacionado con la búsqueda del objeto, las palabras evocan conceptos sin ningún tipo de asociación funcional o instrumental. Bickerton (1990: 184) ofrece un claro ejemplo de ello: el control del fuego por parte del Homo Erectus sólo pudo darse mediante un tratamiento conceptual que retrasa la evocación directa del fuego, de modo que es posible "observar objetivamente cosas que en otras circunstancias podrían despertar emociones demasiado violentas para ser controladas". Esto es, sería difícil imaginar que el fuego fuera usado por una especie carente de un sistema representacional que permitiera pensar sobre él, eliminando así las temibles implicaciones de tal elemento.

Todo lo señalado conduce a un rasgo crucial que permite diferenciar claramente entre palabras por un lado y etiquetas para conceptos u objetos por otro: las palabras liberan a los conceptos de su dependencia con respecto a la percepción sensorial (Bickerton, 1990: 5051; Györi, 1995: 120). Sin embargo, tal rasgo no se aplica a las supuestas palabras usadas ante Rico, que son etiquetas para objetos reales, y que no pueden desvincularse del aquí y el ahora, de igual modo en que las llamadas animales también indican cosas "aquí y ahora" (cfr. Hauser, 2000: 267); esto es, son representaciones de objetos directamente ligadas a la percepción. Es cierto que los elementos implicados en el entrenamiento de algunos animales no humanos no necesitan ser visibles en todas las fases del experimento, pero no lo es menos que necesitan ser perceptibles al menos en algunas (cfr. Bickerton, 1990: 207). De este modo, la diferencia entre las etiquetas animales y las palabras humanas es la que existe entre un conocimiento directamente ligado a la experiencia en los animales, frente a un conocimiento posible incluso en ausencia de experiencia en los humanos. De hecho, podemos concebir conceptos abstractos (como alma, suerte, deshonor, etc.) o incluso fantásticos, irreales

7 Bickerton (1990) analiza la capacidad de las lenguas para disociar eventos que la realidad muestra fusionados, como un todo. Según este autor, tipos de palabras como sustantivos, adjetivos o verbos permiten dividir eventos amalgamados en entidades que los ocasionan, cualidades de tales entidades y acciones efectuadas o sufridas por las entidades. Si tenemos en cuenta esta capacidad de las palabras para separar situaciones en sus diferentes partes constituyentes, no sorprende que las palabras nos permitan evocar predadores y el peligro que representan de una manera separada, o evocar también separadamente un objeto y su recogida. 
(hada, marciano, fantasma, etc.), así como podemos pensar sobre un objeto cotidiano (cama, coche, carne, etc.). En nuestro caso, podemos ir de la palabra al concepto, sin necesidad de percibir el objeto que es el referente para el concepto (de hecho, como hemos señalado, no pocas veces no existe tal referente real). Como señala Deacon (1997: 21), "We inhabit a world full of abstractions, impossibilities, and paradoxes". El siguiente comentario de Györi (1995: 122) sobre los vervets caracteriza certeramente la diferencia apuntada:

A vervet monkey can of course acquire knowledge of an approaching snake without directly experiencing the danger, only by the way of the warning call for snake by one of its conspecifics. But it cannot acquire this knowledge only by communication and without having previous experience with a snake and the danger it means. The communicative signs simply activate the knowledge that the animal possesses either genetically or by individual experience.

A nuestro juicio, tales palabras se ajustan perfectamente al modelo de conocimiento mostrado por Rico. Los signos comunicativos que provocan la búsqueda y recogida de objetos en Rico (verdaderas palabras para nosotros, pero etiquetas para Rico) activan el conocimiento que el perro ha alcanzado en su experiencia individual mediante su entrenamiento doméstico, comprobada posteriormente por Kaminski et alii (2004).

Sin embargo, no se puede decir lo mismo de la adquisición de palabras en los niños. Sobre la base de que pueden aprender asociaciones entre palabras y objetos (como si las palabras fueran etiquetas ligadas a cosas del mundo) en los primeros estadios de desarrollo, los niños no aprenden en un contexto de entrenamiento dirigido a cumplir tareas específicas. De hecho, ni siquiera es necesario que las palabras se asocien a un contexto de etiquetación para que la adquisición sea exitosa: Bloom (2000: 6) señala que "words can be learned without a strict spatial and temporal coocurrence between the word and the meaning", existiendo sobre un $30 \%$ de casos en los que no se produce ninguna co-ocurrencia ${ }^{8}$.

En resumen, los niños aprenden palabras para situarse en el mundo, mientras que Rico sólo reconoce etiquetas para buscar objetos, las reconoce en relación a un propósito funcional, y siempre parece ser así. Por ello, las palabras, libres de la tiranía de la percepción, no pueden ser asimiladas a etiquetas para conceptos, como lo muestra el que nos permitan imaginar todo tipo de entidades irreales. Las palabras son elementos mucho más abstractos que las etiquetas.

Esta naturaleza abstracta es defendida de modo convincente por la teoría de la referencia simbólica de Deacon $(1997,2003)$. Según Deacon (1997: 54), la creencia usual (no técnica) de que las llamadas animales (y esto se puede extender al supuesto uso de palabras por animales) son como palabras supone malentender la noción de referencia. Mientras la referencia de tipo no simbólico (ilustrada por las llamadas de alarma y por el uso de etiquetas en Rico) es directa, incluso aunque esté mediada por un concepto, la de tipo simbólico es indirecta, construida sobre un sistema complejo de relaciones en forma de redes interconectadas, lo cual va mucho más allá de un mero conjunto de trazados uno a uno entre señal y referente;

8 Los niños ni siquiera precisan de habilidades sensoriales plenas para aprender palabras: como se muestra en referencias como Petitto (1997: 47 y ss.) o Emmorey (2002: cap. 6), entre otras muchas, los niños sordos que adquieren una lengua de signos muestran el mismo tipo de desarrollo que el de los niños oyentes que aprenden modalidades orales del lenguaje. Lo mismo se aplica incluso a los niños ciegos (cfr. Bloom, 2000: 7). 
por ejemplo, del mismo modo en que funciona un Thesaurus, donde una palabra nos remite a otras relacionadas. Pinker y Jackendoff (2005: 214) concuerdan con tal visión:

Another hallmark of words is that their meanings are defined not just by the relation of the word to a concept but by the relation of the word to other words in the lexicon, forming organized sets such as superordinates, antonyms, meronyms (parts) [...].

Así, la estructura de los símbolos, representada paradigmáticamente por las palabras, difiere cualitativamente de la de las llamadas animales. Nuestras palabras son elementos abstractos, desligados del mundo real y conectados entre sí de manera abstracta: las palabras tienen un significado potencial que debe ser realizado mediante un contexto concreto de referencia (Deacon, 2003).

Usaremos la habilidad numérica en animales para ilustrar la postura de Deacon. Como muestran varias referencias (Carey, 2004; Dehaene, 1997; Gallistel, 1990 o Hauser, 2000, entre otras), bastantes especies tienen un sentido numérico básico con valor adaptativo, que parece ir más allá de una mera capacidad de percepción global o subitizing, si bien esto no implica que los animales tengan el mismo nivel de entendimiento numérico que el poseído por los humanos. Para explicar tal diferencia, Hauser (2000: 85) distingue entre categoría numérica y concepto numérico. Este último, frente a la categoría numérica, es un tipo de símbolo mental, y, como sucede con las palabras, guarda una relación particular con otros símbolos numéricos. Es decir, según Hauser, señalar un montón de galletas y decir ' 7 ' puede indicar que se conoce el hecho de que hay 7 galletas, pero saber que 7 equivale a $8-1$, o a $4+3$ es algo bien diferente. Por ello, aunque variadas especies tienen categorías numéricas, equivalentes a etiquetas, carecen de conceptos numéricos, que requieren, como las palabras, una interrelación abstracta (si bien algunos trabajos sugieren que los chimpancés entienden conceptos aditivos y sustractivos, estos se restringen a números pequeños; cfr. al respecto Biro y Matsuzawa, 2001 y Boysen y Hallberg, 2000).

En conclusión, lo que Rico ha aprendido realmente parece ser la asociación entre etiquetas y objetos/conceptos. Esas asociaciones dependen de la percepción de Rico y de su experiencia individual en el contexto de un altamente específico y rígido procedimiento de búsqueda de objetos. No hay, pues, evidencia de que Rico posea verdaderas palabras.

\subsection{Implicaciones del fast mapping}

En el apartado 2 señalamos que el segundo experimento diseñado por Kaminski et alii (2004) pretendía verificar la capacidad de Rico para establecer nuevas asociaciones entre palabras y objetos sobre la base de una única exposición (fast mapping). El éxito del perro en el experimento condujo a que los autores sugirieran que Rico posee la habilidad de aprender por exclusión y que adquirió el principio de que los objetos tienen etiquetas. ¿Cómo casa esta habilidad con la discusión efectuada en el anterior subapartado? En concreto, ¿implican esos logros de Rico la incorrección de nuestro enfoque? Creemos que no. El aprendizaje por exclusión se inserta plenamente en el tipo de pensamiento on-line, modelo característico, según Bickerton (1995), del pensamiento animal.

El pensamiento on-line "involves computations carried out only in terms of neural responses elicited by the presence of external objects" (Bickerton, 1995: 90). El segundo 
tipo de pensamiento sugerido por este autor, off-line, es muy diferente, ya que "involves computations carried out on more lasting internal representations of those objects", de modo que "new information could be processed without needing to be triggered by environmental input and without invoking immediate behavioural consequences" (Bickerton, 1995: 59). A la luz de ambas definiciones, el aprendizaje por exclusión de Rico no se aparta del modelo on-line propio del reino animal.

Por otro lado, ni siquiera pensamos que el aprendizaje por exclusión sea una habilidad digna de especial consideración en lo que respecta al supuesto aprendizaje de palabras por parte de Rico; más bien, tal aprendizaje sugiere, en nuestra opinión, un subtipo del mecanismo general de formación de asociaciones. Si así fuera, el aprendizaje por exclusión sería un ejemplo del nivel 2 de inteligencia de MacPhail $(1982,1987)$. Mientras el nivel 1 permite formar asociaciones entre un estímulo y una respuesta, el nivel 2, propio de casi todos los vertebrados, permite asociar un estímulo con otro estímulo, de modo que el organismo puede formar expectativas (MacPhail, 1987: 651). Según este autor, el mecanismo de formación de asociaciones "lies at the core of problem-solving in nonhumans" (MacPhail, 1987: 654), posibilitando "the successful detection of causal links between events" (MacPhail, 1987: 645). Creemos que esto es exactamente lo que sucede en el aprendizaje por exclusión de Rico. Si tenemos en cuenta las asociaciones previas y bien establecidas (recuérdese que Rico acertó en 37 de los 40 intentos) entre las 'palabras' aprendidas por Rico y los objetos a los que se refieren, no debería sorprender que, durante las pruebas con nuevos elementos, Rico seleccionara el único objeto que no rompía una asociación previamente conocida.

Por tanto, aprender por exclusión no prueba que Rico use verdaderas palabras; de hecho, no prueba más que el uso de etiquetas. Sólo el lenguaje, a través de sus palabras, permite extraer deducciones sobre seres, objetos o clases ausentes. Cuando aprendemos con palabras, aprendemos que aunque éstas pueden evocar objetos, lo que hacen realmente es evocar categorías conceptuales que a menudo carecen de referente en la realidad. Con las palabras, podemos establecer vínculos (como Rico parece haber hecho) con objetos o eventos del mundo (si bien los humanos son conscientes de la mediación conceptual existente en esas relaciones asociativas, con todas las consecuencias señaladas). Además, podemos vincular una palabra con el concepto que evoca o con el objeto evocado, a través, por ejemplo, de una representación pictórica (dibujo, fotografía, etc.) del objeto al que alude la palabra en nuestra lengua: tal representación basta para inferir la relación palabra-concepto-objeto. Finalmente, y sobre todo, aprendemos, como señaló Quine (1960: 31 y ss.), asociando palabras con palabras: si se nos enuncian los rasgos que definen un concepto, podremos establecer el vínculo entre una palabra y el concepto asociado antes de conocer si existe un objeto real al que se refiera el concepto.

Quizás se nos podría objetar que hemos caracterizado la utilidad o el resultado que los humanos han extraído de la adquisición y el uso de palabras, en vez de caracterizar la propia adquisición (una tarea en la que Rico supuestamente habría tenido éxito). Sin embargo, esa objeción es aparente, porque el producto descansa en una configuración específica de los vínculos entre palabras, conceptos y objetos. Esto es, lo que los humanos han adquirido a través del lenguaje (el cual es inseparable de las palabras) ofrece los fundamentos de la existencia de diferentes caminos (como la experiencia directa, las palabras, o bien otros tipos de representaciones) en nuestros procesos de aprendizaje. Por tanto, tal utilidad sería el 
síntoma que revela cómo actúan las palabras en relación a la realidad en general y a nuestro mundo en particular.

Por otro lado, recordemos que, según Kaminski et alii (2004), el aprendizaje de palabras de Rico puede descomponerse en un conjunto de mecanismos más simples: adquisición del principio de que los objetos tienen etiquetas, aprendizaje por exclusión y habilidad de almacenar ese conocimiento en la memoria. De ese modo, los resultados del estudio "strongly support the view that a seemingly complex linguistic skill previously described only in human children may be mediated by simpler cognitive building blocks that are also present in another species" (Kaminski et alii, 2004: 1683), apoyando también que "some of the perceptual and cognitive mechanisms that may mediate the comprehension of speech were already in place before early humans began to talk." (Kaminski et alii, 2004: 1683). Si bien la última asunción es correcta, tal como trataremos, no lo es tanto asumir que "a seemingly complex linguistic skill" como la adquisición de palabras puede reducirse a un conjunto de principios como los atribuidos a Rico.

Usualmente, el habla se ha considerado un rasgo únicamente humano (cfr. Lieberman, 1991, 1998, 2003), pero descubrimientos recientes muestran que animales humanos y no humanos (sobre todo, mamíferos y aves) no sólo comparten capacidades perceptivas que subyacen al habla, sino también rasgos implicados en su producción. Al respecto de ésta, es bien conocido el énfasis de Lieberman en que el tracto vocal tiene una configuración única entre todos los mamíferos: mientras la laringe no humana (y la de los bebés humanos) está muy alta, la humana ocupa una posición más baja. Este hecho anatómico, propio según Lieberman de los humanos adultos, permite producir un gran conjunto de sonidos lingüísticos. Sin embargo, trabajos como Fitch y Reby (2001) o Hauser y Fitch (2003) sostienen que una laringe descendida no es específicamente humana. De hecho, Hauser y Fitch (2003) señalan que las vocalizaciones de animales tan diversos como cerdos, perros, cabras, leones o koalas, se basan en una laringe móvil, que desciende durante las vocalizaciones y luego retorna a su posición. Pero ni siquiera una laringe descendida de manera permanente es un rasgo únicamente humano: Fitch y Reby (2001) muestran claramente que los machos de gamos y ciervos rojos tienen también una laringe permanentemente descendida comparable a la posición de la laringe humana adulta, y que la mueven incluso más abajo durante sus vocalizaciones.

Del mismo modo, los mecanismos responsables de la percepción del habla tampoco son específicamente humanos, ni específicos del habla (cfr. Hauser, 2001 y Hauser y Fitch, 2003). Por ejemplo, la capacidad de dividir un continuum de sonidos del habla en categorías discretas es compartida con mamíferos y aves, por lo que parece apuntar a un mecanismo auditivo general. Además, algunos animales perciben, como nosotros, clases de equivalencia acústica: Hauser (2001) muestra que los monos tamarindos pueden discriminar entre oraciones holandesas y japonesas. Por otro lado, varias especies de monos comparten con nosotros mecanismos de aprendizaje estadístico, que se basan en la posibilidad de coocurrencia entre sonidos y que permiten deducir los lindes entre palabras a partir del continuum de habla. En resumen, "humans share with other animals the core mechanisms for speech perception" (Hauser, 2001: 428) (cfr. también Hauser, Weiss y Marcus, 2002 como ejemplo de un estudio concreto).

Sin embargo, la continuidad básica entre animales humanos y no humanos al respecto de los mecanismos de comprensión y producción del habla se restringe a capacidades generales 
de vocalización y de recepción, y no a capacidades específicamente lingüísticas. Dicho en otros términos: es cierto que algunos requisitos necesarios para adquirir el lenguaje están presentes en otros animales, pero esto no implica obviamente que, en ellos, esos requisitos se relacionen directamente con funciones específicamente lingüísticas ni se traduzcan en tales funciones. Por tanto, como hemos intentado mostrar, parece exagerado deducir que los mecanismos en los que se puede descomponer el fast mapping dan cuenta automáticamente de la adquisición de palabras en un sentido técnico: para adquirirlas, se necesita más que los tres principios que Kaminski et alii (2004) atribuyen a Rico. Es indudable que debe existir un conjunto de condiciones iniciales requeridas para que pueda darse la adquisición de palabras, pero esto en sí mismo no basta. Parece razonable sostener que procesos mentales desconocidos en los animales surgieron en nuestra especie y complementaron a los principios antes referidos, dando así lugar a los procesos simbólicos humanos, representados paradigmáticamente por las palabras (cfr. Chomsky, Hauser y Fitch, 2002: 1576 o Hauser, 2000: 267 sobre las dificultades que existen en trazar antecedentes evolutivos de las palabras en animales no humanos). En suma, al igual que sucede con la sintaxis y su propiedad de recursión, las palabras humanas parecen estar fuera del alcance de Rico, y lo mismo rige para el conjunto de supuestas 'palabras' animales.

Un último aspecto que deseamos señalar es el siguiente: como se habrá percibido a lo largo del presente trabajo, hemos limitado conscientemente nuestra argumentación al ámbito estrictamente semántico o significativo de las palabras. Es obvio, sin embargo, que estas unidades tienen otra dimensión, quizás incluso más característica, la sintáctica, representada por los aspectos gramaticales inherentes a ellas. A pesar de la operatividad de la división entre un léxico y un sistema computacional o sintaxis, dotado de la propiedad de recursión, el léxico (en sentido pleno) configura o determina las agrupaciones sintácticas, teniendo en cuenta las propiedades de subcategorización de cada una de las piezas léxicas, que permiten su combinación con otras piezas diferentes. En este sentido, son relevantes los variados mecanismos o rasgos gramaticales de las palabras (concordancia, caso, etc.), desarrollados de manera temprana en los niños, pero que se hallan ausentes por completo en animales no humanos. Desde tal perspectiva, las diferencias, que no siempre se han tenido en cuenta ${ }^{9}$, todavía se agrandarían más: señala Anderson (2004: 292-293) en su análisis de los importantes resultados obtenidos por el bonobo Kanzi (el animal no humano cuyos logros no tienen precedentes en la cognición animal), que aunque es capaz de desarrollar una representación del significado en la que asocia participantes de la oración y roles temáticos, cuando la interpretación depende de elementos gramaticales, los resultados de Kanzi son muy pobres, de modo que tales elementos con significado gramatical son ignorados. Por tanto, como señalábamos, desde la perspectiva gramatical, ni siquiera habría discusión posible. De ahí que hayamos optado por limitarnos al ámbito semántico, asumiendo que no es necesario ni siquiera salir de él para mostrar que los animales carecen de palabras o de equivalentes de ellas en un sentido no trivial.

9 A este respecto, numerosos experimentos que trataban de enseñar el American Sign Language a primates asumían que estos animales eran capaces de producir palabras en la modalidad gestual del lenguaje, cuando en realidad a tales supuestas 'palabras' les faltaba todo tipo de elementos gramaticales, los cuales, sin embargo. están presentes en las lenguas de signos. 


\section{Conclusiones}

El principal objetivo del artículo ha sido mostrar que, desde una perspectiva lingüística, poseer etiquetas (para objetos o conceptos) no equivale a poseer palabras, por lo que ambos tipos de elementos no deberían ser asimilados ni igualados. No ha sido nuestra intención restar importancia a los hallazgos de Kaminski et alii (2004) al respecto de la habilidad de fast mapping de Rico. Más bien, hemos expresado nuestras dudas sobre el hecho de que el fast mapping deba ser identificado con la adquisición de palabras, si estas unidades se entienden en un sentido técnico estricto. Tal postura, en todo caso, no implica de ningún modo un apriorismo: como escribe Anderson (2004: 36)

If it turns out that either naturally occurring communicative behavior or abilities induced in laboratory experiments indeed have the characteristic properties of a human language, linguists in general will be fascinated, not repelled, by that fact.

Como sucede con un niño, Rico puede aprender, tras una sola exposición, el camino que va del modelo acústico arbitrario al objeto que evoca ese modelo. Sin embargo, el niño forma hipótesis sobre el significado de la nueva palabra, mientras que Rico forma hipótesis sobre la etiqueta que le conduce a identificar y traer el objeto concreto. Es cierto que las etiquetas que Rico ha aprendido son palabras de (y en) un código lingüístico, pero esto no implica que sean verdaderas palabras para Rico. El valor de las palabras como tales (la conformación cognitiva y el resultado cognitivo inherentes a ellas) sobrepasa la tarea de identificar áreas de la realidad, permitiendo no sólo configurar tal realidad, sino también poder imaginar aspectos inexistentes en ella: "We create worlds with language" (Jerison, 1985: 31).

En el proceso de identificación que permite a Rico encontrar objetos concretos, las palabras (que son símbolos, después de todo) están únicamente dotadas de una capacidad de referencia genérica (evocar un objeto real diferente de la señal que evoca, lo que supone según Deacon una referencia directa), pero las 'palabras' de Rico carecen de los rasgos centrales discutidos en el trabajo, que se pueden resumir en la naturaleza indirecta de la referencia simbólica. A este respecto, sería interesante verificar si otros elementos sin la estructura cognitiva específica de las palabras podrían haber arrojado el mismo resultado en Rico. Si así fuera, el aspecto relevante en la habilidad de Rico sería su competencia para reconocer y almacenar las evocaciones que algunos elementos sugieren con respecto a algunos objetos. Pero este proceso de sustitución no podría ser desarrollado en sentido inverso: ningún elemento con referencia directa podría satisfacer todas las funciones que las palabras tienen en las lenguas y entre sus usuarios.

Ese es el factor clave a nuestro juicio para determinar si las habilidades de fast mapping de Rico (de las que no dudamos) capacitan a Rico para aprender palabras humanas, como Kaminski et alii (2004) sugieren. Es claro que el fast mapping juega un papel relevante en la adquisición de palabras en el niño, pero tal habilidad no es suficiente para adquirir palabras. Como ha sucedido en relación a otras capacidades que subyacen a habilidades lingüísticas muy posteriores evolutivamente (cfr. 3.3.), la presencia de tales capacidades en animales no humanos no implica que se usen del mismo modo que en nosotros, ni que se alcance el mismo resultado que los humanos han alcanzado. Eso no significa que neguemos que dis- 
tintos animales hayan evolucionado de diferentes modos y que algunos se hayan aproximado a sistemas que recuerdan a (pero que no son) palabras. En este sentido, como señalábamos al principio de este trabajo, nos volvemos a declarar contrarios a la tradición progresivista. Añadimos así, en suma, nuestros comentarios a otros hechos a Kaminski et alii $(2004)^{10}$ para enfatizar los interesantes hallazgos y problemas que el caso pone sobre el tapete.

\section{Referencias bibliográficas}

Anderson, S.R. (2004): Doctor Dolittle's delusion. Animals and the uniqueness of human language. New Haven, Yale University Press.

Bickerton, D. (1990): Language and species. Chicago, Chicago University Press. Citamos por la trad. de M.A. Valladares, Lenguaje y especies. Madrid, Alianza, 1994.

Bickerton, D. (1995): Language and human behavior. Seattle, University of Washington Press.

Biro, D. y T. Matsuzawa (2001): "Chimpanzees numerical competence: Cardinal and ordinal skills".

En Matsuzawa, T. (ed.): Primate origins of human cognition and behavior. Nueva York, Springer-

Verlag, págs. 199-225.

Bloom, P. (2000): How children learn the meanings of words. Cambridge, MA, MIT Press.

Bloom, P. (2004): "Can a dog learn a word?". Science, 304, págs. 1605-1606.

Boysen, S.T. y K.I. Hallberg (2000): "Primate numerical competence: Contributions toward understanding nonhuman cognition". Cognitive Science, 24/3, págs. 423-443.

Budiansky, S. (1998): If a lion could talk. Animal intelligence and the evolution of consciousness.

Nueva York, Free Press. Hay trad. de B. Martín, Si los animales hablaran...no les entenderiamos.

La evolución de la conciencia y la inteligencia. Madrid, Ateles, 2001.

Carey, S. (1978): “The child as word-learner". En Halle, M., J. Bresnan y G. Miller (eds.): Linguistic theory and psychological reality. Cambridge, MA, MIT Press, págs. 264-293.

Carey, S. (2004): "On the origin of concepts". Daedalus, 133/1, págs. 59-68.

Cheney, D. y R. Seyfarth (1990): How monkeys see the world. Chicago, University of Chicago Press.

Cheney, D. y R. Seyfarth (1999): "Mechanisms underlying the vocalizations of nonhuman primates".

En Hauser, M.D. y M. Konishi (eds.): The design of animal communication. Cambridge, MA, MIT Press, págs. 629-644.

Christiansen, M. y S. Kirby (eds.) (2003): Language evolution. Nueva York, Oxford University Press.

Deacon, T. (1997): The symbolic species. The co-evolution of language and the human brain. London, Penguin.

Deacon, T. (2003): "Universal Grammar and semiotic constraints". En Christiansen, M. y S. Kirby (eds.), págs. 111-139.

Dehaene, S. (1997): The number sense: How the mind creates mathematics. Oxford, Oxford University Press.

Dennett, D.C. (1995): Darwin's dangerous idea. Nueva York, Simon y Schuster. Citamos por la trad. de C. Pera, La peligrosa idea de Darwin. Barcelona, Galaxia Gutenberg / Círculo de Lectores, 1999.

10 Para decidir si Rico puede adquirir palabras, Bloom (2004: 1606) propone nuevos experimentos que puedan responder a preguntas como las siguientes: "Can Rico learn a new word by being shown an object and hearing a person name it? Can he learn a word for something other than a small fetchable object? Can he display knowledge of a word in some way other than fetching? [... Can Rico follow an instruction not to fetch an item, just as one can tell a child not to touch something?". 
Downes, S. (2003): "Baldwin effects and the expansion of explanatory repertoire in Evolutionary Biology". En Weber, B. y D. Depew (eds.): Evolution and learning. The Baldwin effect reconsidered. Cambridge, MA, MIT Press, págs. 33-51.

Emmorey, K. (2002): Language, cognition, and the brain. Insights from Sign Language research. Mahwah, NJ, Lawrence Erlbaum.

Evans, C.S. y L. Evans (1999): "Chicken food calls are functionally referential". Animal Behaviour, 58, págs. $307-319$.

Fitch, W.T. y D. Reby (2001): "The descended larynx is not uniquely human". Proceedings of the Royal Society of London, 268, págs. 1669-1675.

Fouts, R. (1997): Next of kin. What chimpanzees have taught me about who we are. Nueva York, Morrow.

Gallistel, C.R. (1990): The organization of learning. Cambridge, MA, MIT Press.

Györi, G. (1995): "Animal communication and human language: searching for their evolutionary relationship". En Puppel, S. (ed.): The biology of language. Amsterdam y Philadelphia, John Benjamins, págs. 99-126.

Hauser, M.D. (2000): Wild minds. What animals really think. Nueva York, Henry Holt. Citamos por la trad. de A. Herrera, Mentes salvajes. ¿Qué piensan los animales? Buenos Aires, Granica, 2002.

Hauser, M.D. (2001): “What's so special about speech?". En Dupoux, E. (ed.): Language, brain, and cognitive development. Essays in honor of Jacques Mehler. Cambridge, MA, MIT Press, págs. $417-433$.

Hauser, M.D., N. Chomsky y W.T. Fitch (2002): "The faculty of language: What is it, who has it, and how did it evolve?". Science, 298, págs. 1569-1579.

Hauser, M.D. y W.T. Fitch (2003): "What are the uniquely human components of the language faculty?". En Christiansen, M. y S. Kirby (eds.), págs. 158-181.

Hauser, M.D., D. Weiss y G. Marcus (2002): "Rule learning by cotton-top tamarins". Cognition, 86/1, págs. B15-B22.

Herman, L., D. Richards y J. Wolz (1984): "Comprehension of sentences by bottlenosed dolphins". Cognition, 16, págs. 129-219.

Hölldobler, B. y E.O. Wilson (1994): Journey to the ants. A story of scientific exploration. Cambridge, MA, Harvard University Press. Hay trad. de J. Ros, Viaje a las hormigas. Una historia de exploración cientifica. Barcelona, Crítica, 1996.

Jerison, J. (1985): “Animal intelligence as encephalization”. En Weiskrantz, L. (ed.): Animal intelligence. Oxford, Clarendon Press, págs. 21-35.

Kaminski, J., J. Call y J. Fisher (2004): "Word learning in a domestic dog: Evidence for 'fast mapping". Science, 304, págs. 1682-1683.

Lieberman, P. (1991): Uniquely human. The evolution of speech, thought, and selfless behavior. Cambridge, MA, Harvard University Press.

Lieberman, P. (1998): Eve spoke. Human language and human evolution. Nueva York, W.W. Norton.

Lieberman, P. (2003): "Motor control, speech, and the evolution of human language". En Christiansen, M. y S. Kirby (eds.), págs. 255-271.

MacPhail, E. (1982): Brain and intelligence in vertebrates. Oxford, Clarendon Press.

MacPhail, E. (1987): "The comparative psychology of intelligence". Behavioral and Brain Sciences, 10 , págs. 645-656.

Marler, P. (1977): “The structure of animal communication sounds". En Bullock, T. (ed.): Recognition of complex acoustic signals. Berlín, Springer-Verlag, págs. 17-35.

Mitani, J.C. y P. Marler (1989): "A phonological analysis of male gibbon singing behavior". Behavior, 109 , págs. $20-45$.

Owings, D. y E. Morton (1998): Animal vocal communication: A new approach. Cambridge, Cambridge University Press. 
Patterson, D.K. e I.M. Pepperberg (1994): "A comparative study of human and parrot phonation: I. Acoustic and articulatory correlates of vowels". Journal of the Acoustic Society of America, 96 , págs. 634-648.

Patterson, D.K. e I.M. Pepperberg (1998): "A comparative study of human and Grey parrot phonation: Acoustic and articulatory correlates of stop consonants". Journal of the Acoustic Society of America, 103, págs. 2197-2213.

Pepperberg, I.M. (1999): The Alex studies. Cognitive and communicative abilities of grey parrots. Cambridge, MA, Harvard University Press.

Pepperberg, I.M. (2004): "Evolution of communication from an avian perspective". En Kimbrough Oller, D. y U. Griebel (eds.): Evolution of communication systems. A comparative approach. Cambridge, MA, MIT Press, págs. 171-192.

Petitto, L.A. (1997): "In the beginning: On the genetic and environmental factors that make early language acquisition possible". En Gopnik, M. (ed.): The inheritance and innateness of grammars. Nueva York, Oxford University Press, págs. 45-69.

Pinker, S. y R. Jackendoff (2005): "The faculty of language: What's special about it?". Cognition, 95/2, págs. 201-236.

Premack, D. (1970): "Functional analysis of language". Journal of the Experimental Analysis of Behavior, 14, págs. 107-125.

Quine, W.V.O. (1960): Word and object. Cambridge, MA, MIT Press. Citamos por la trad. de M. Sacristản, Palabra y objeto. Barcelona, Herder, 2001.

Rondal, J.A. (2000): Le langage: de l'animal aux origines du langage humain. Sprimont, Mardaga.

Saussure, F. de (1916): Cours de linguistique genérale. Paris, Payot. [Ed. por C. Bally y A. Sechehaye]. Citamos por la trad. de M. Armiño, Curso de linguiistica general. Madrid, Akal, 1980.

Savage-Rumbaugh, S., S.G. Shanker y T.J. Taylor (1998): Apes, language, and the human mind. Oxford, Oxford University Press.

Seyfarth, R., D. Cheney y P. Marler (1980a): "Monkey responses to three different alarm calls: evidence of predator classification and semantic communication". Science, 210, págs. 801-803.

Seyfarth, R., D. Cheney y P. Marler (1980b): "Vervet monkeys alarm calls: semantic communication in a free-ranging primate". Animal Behaviourr, 28, págs. 1070-1094.

Spelke, E. (2003): "What makes us smart? Core knowledge and natural language". En Gentner, D. y S. Goldin-Meadow (eds.): Language in mind. Advances in the study of language and thought. Cambridge, MA, MIT Press, págs. 277-311.

Tomasello, M. (2003): Constructing a language. A usage-based theory of language acquisition. Cambridge, MA, Harvard University Press.

Tomasello, M. y J. Call (1997): Primate cognition. Oxford, Oxford University Press.

Zuberbühler, K. (2002): "A syntactic rule in forest monkey communication". Animal Behaviour, 63, págs. 293-299. 